\title{
Identifying the Location in the Host Galaxy of Short GRB 111117A with the Chandra Sub-arcsecond Position
}

T. Sakamoto ${ }^{*}, b$ E. Troja, ${ }^{c}$ K. Aoki, ${ }^{d}$ S. Guiriec, ${ }^{c}$ M. Im, ${ }^{e}$ G. Leloudas, ${ }^{f, g}$ D. Malesani, ${ }^{g}$ A. Melandri, ${ }^{h}$ A. de Ugarte Postigo, ${ }^{i, g}$ Y. Urata ${ }^{j}$ and D. Xu ${ }^{k}$

${ }^{a}$ CRESST and Astroparticle Physics laboratory, NASA/GSFC, Greenbelt, MD 20771, U.S.A.

${ }^{b}$ Joint Center for Astrophysics, University of Maryland, Baltimore County, 1000 Hilltop Circle, Baltimore, MD 21250, U.S.A.

${ }^{c}$ NASA Postdoctoral Program Fellow, Goddard Space Flight Center, Greenbelt, MD 20771, U.S.A.

${ }^{d}$ Subaru Telescope, National Astronomical Observatory of Japan, 650 North A'ohoku Place, Hilo, HI 96720

${ }^{e}$ Center for the Exploration of the Origin of the Universe (CEOU), Department of Physics and Astronomy, Seoul National University, Seoul, 151-747, Korea

${ }^{f}$ The Oskar Klein Centre, Department of Physics, Stockholm University, 10691 Stockholm, Sweden

${ }^{g}$ Dark Cosmology Centre, Niels Bohr Institute, University of Copenhagen, Juliane Maries Vej 30, 2100 Copenhagen $\emptyset$, Denmark

${ }^{h}$ INAF - Osservatorio Astronomico di Brera, via Bianchi 46, I-23807 Merate (LC), Italy

${ }^{i}$ Instituto de Astrofísica de Andalucía (CSIC), Glorieta de la Astronomía s/n, 18008 Granada, Spain

${ }^{j}$ Institute of Astornomy, National Central University, Chung-Li 32054, Taiwan

${ }^{k}$ Department of Particle Physics and Astronomy, The Weizmann Institute of Science, Rehovot 76100, Israel

\section{E-mail: Taka.Sakamoto@nasa.gov}

We present our successful program using Chandra for identifying the X-ray afterglow with subarcsecond accuracy for the short GRB 111117A discovered by Swift and Fermi. Thanks to our rapid target of opportunity request, Chandra clearly detected the X-ray afterglow, whereas no optical afterglow was found in deep optical observations. Instead, we clearly detect the host galaxy in optical and also in near-infrared bands. We found that the best fit photometric redshift of the host is $z=1.31_{-0.23}^{+0.46}$ (90\% confidence), making it one of the highest redshift short GRBs. Furthermore, we see an offset of $1.0 \pm 0.2$ arcseconds, which corresponds to $8.4 \pm 1.7 \mathrm{kpc}$ assuming $\mathrm{z}=1.31$, between the host and the afterglow position. We discuss the importance of using Chandra for obtaining sub-arcsecond localization of the afterglow in X-rays for short GRBs to study GRB environments in great detail.

Gamma-Ray Bursts 2012 Conference -GRB2012,

May 07-11, 2012

Munich, Germany

${ }^{*}$ Speaker. 


\section{Introduction}

Gamma-ray bursts (GRBs) are traditionally divided in two classes based on their duration and spectral hardness: the long duration/soft spectrum GRBs, and the short duration/hard spectrum GRBs [1]. The long standing paradigm is that these two phenomenological classes of GRBs originate from different progenitor systems. A preponderance of evidence now links long GRBs with the death of massive stars [2], yet the origin of short GRBs remains largely unknown. The common notion that short bursts originate from coalescing compact binaries, either neutron star-neutron star (NS-NS) or neutron star-black hole (NS-BH) mergers [3, 4], makes them the most promising tool to aid in the direct detection of gravitational waves (GWs).

We report the first results of our Chandra program which led to the accurate localization of GRB 111117A detected by Swift and Fermi. Our results were leveraged with an intense groundbased follow-up campaign. No optical/infrared counterpart was found, therefore our Chandra localization uniquely provides the most accurate sub-arcsecond position.

\section{Prompt Emission}

The light curve of the prompt emission is composed of two episodes: the first episode shows multiple overlapping pulses with a total duration of $0.3 \mathrm{~s}$, and the second episode is composed of two pulses with a duration of $0.1 \mathrm{~s}$ (Figure 1). The duration is $T_{90}=464 \pm 54 \mathrm{~ms}$ ( $1 \sigma$ error; $15-350$ $\mathrm{keV})$ and $T_{90}=512 \pm 181 \mathrm{~ms}(1 \sigma$ error; $50-300 \mathrm{keV})$ measured by BAT and GBM respectively. This $T_{90}$ duration is significantly shorter than $2 \mathrm{~s}$, which is the standard classification of short GRBs in the BATSE GRBs [1]. Furthermore, this duration is shorter than $0.7 \mathrm{~s}$, which is claimed to be the dividing line between long and short GRBs for the Swift sample [5]. The spectral lag between the $100-350 \mathrm{keV}$ and the $25-50 \mathrm{keV}$ band is $0.6 \pm 2.4 \mathrm{~ms}$, which is consistent with zero. The timeintegrated spectral properties are investigated by performing a joint spectral analysis with BAT and GBM data. The spectrum is extracted from $t_{0, \mathrm{BAT}}+0.024 \mathrm{~s}$ to $t_{0, \mathrm{BAT}}+0.520 \mathrm{~s}$. We find that a power-law multiplied by an exponential cutoff provides the best representative model of the data. The best fit parameters in this model are the power-law photon index $\alpha^{\mathrm{CPL}}=-0.28_{-0.26}^{+0.31}$ and $\mathrm{E}_{\text {peak }}$ $=420_{-110}^{+170} \mathrm{keV}$.

\section{Afterglow}

The Swift XRT started GRB 111117A observation $76.8 \mathrm{~s}$ after the trigger. A fading X-ray source was found within the BAT error circle. The Chandra observation (a total exposure time of $19.8 \mathrm{ks}$ ) started $\sim 3$ days after the trigger. The X-ray afterglow is clearly detected in the Chandra observation with $3.9 \sigma$ significance within the XRT error circle. The combined Swift XRT and Chandra X-ray afterglow light curve is well fit by a simple power-law with the index of $-1.25_{-0.12}^{+0.09}$. As shown in Figure 2, the X-ray afterglow of GRB 111117A belongs to a dim population of the Swift short GRBs. The spectrum collected in the Photon Counting (PC) mode is well described by an absorbed power-law model. The best fit spectral parameters are a photon index of $-2.19_{-0.38}^{+0.36}$ and an excess $N_{H}$ of $1.8_{-1.0}^{+1.1} \times 10^{21} \mathrm{~cm}^{-2}$ assuming the galactic $N_{H}$ at the burst direction of $3.7 \times$ $10^{20} \mathrm{~cm}^{-2}[6]$. 


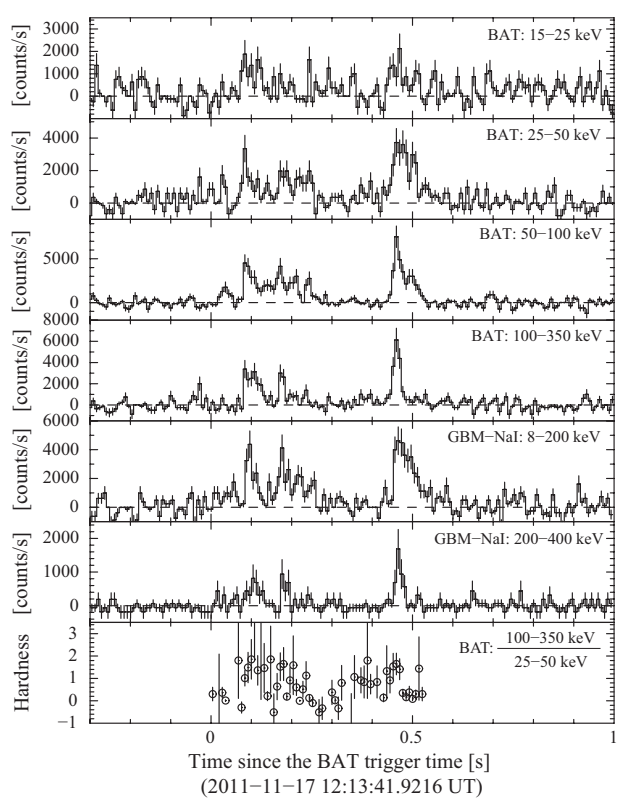

Figure 1: The background subtracted $5 \mathrm{~ms}$ light curves of Swift BAT and Fermi GBM. The bottom panel shows the hardness ratio between the 100$350 \mathrm{keV}$ and the $25-50 \mathrm{keV}$ of the BAT data.

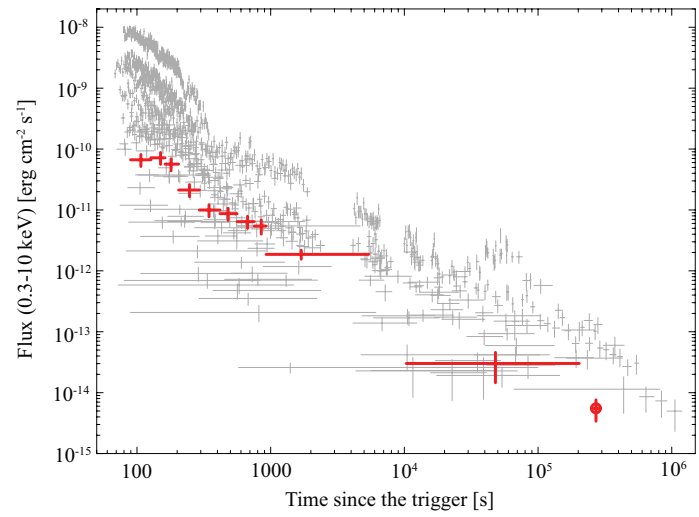

Figure 2: Comparison of X-ray afterglow light curves of short GRBs observed by Swift XRT (gray) and GRB 111117A (red). The Chandra data point of GRB 111117A is shown in red filled circle.

We investigate the possible optical afterglow emission by using the image subtraction technique between the early and the late time epoch observations by Telescopio Nazionale Galileo (TNG) and Gran Telescopio CANARIAS (GTC). We find no significant emission at the Chandra $\mathrm{X}$-ray afterglow location in the subtracted images in both the TNG and the GTC observations.

The optical-to-X-ray spectral index $\beta_{\mathrm{OX}}[7]$ is estimated to be $\lesssim 0.78$ using the X-ray flux density measured at 11 hours after the burst at $3 \mathrm{keV}$, and the optical afterglow limit based on the GTC $r$ band. This upper limit of $\beta_{\mathrm{OX}}$ is within the allowed range of the standard afterglow model between 0.5 to 1.25 .

\section{Host Galaxy}

The host galaxy of GRB 111117A has been detected from the near infrared to the optical bands. There is only one near-infrared and optical source located near the Chandra X-ray afterglow position. Although the weak nature of the source makes it difficult to investigate whether the source is extended or not, the optical flux of the source is constant between 7 hours and 14 days after the burst at a level of $\sim 1.1 \mu \mathrm{Jy}$. Therefore, we conclude that the source detected in $K, J, z, i, r, g$, and $R$ bands is the host galaxy of GRB 111117A (Figure 3).

To estimate the redshift of the host galaxy, we perform a spectral energy density (SED) fit with the stellar population model of [8]. We find the best estimated redshift to be 1.31 (90\% confidence interval $1.08<z_{p h}<1.77$ ). The likelihood that the redshift is correct is $80 \%$ (reduced $\chi^{2}$ of the fit is 0.65 ). The best fit SED template is the case with the luminosity weighted mean stellar age of 0.1 Gyr and a mass of $\sim 1 \times 10^{9} \mathrm{M}_{\odot}$ (Figure 4). 


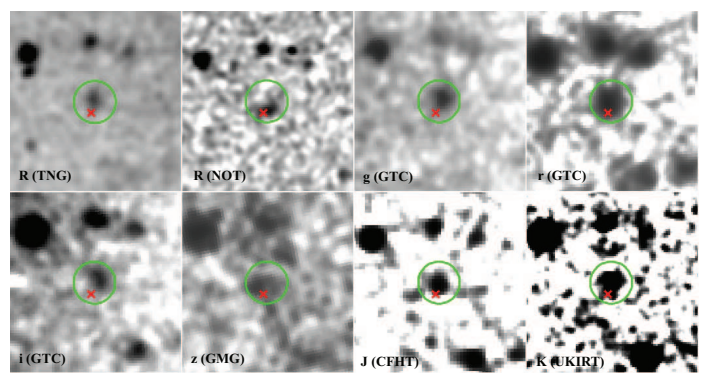

Figure 3: Multi-color images at the field of GRB 111117A. From left to right, and top to bottom, the images are TNG $R$, NOT $R$, GTC $g$, GTC $r$, GTC $i$, GMG $z$, CFHT $J$ and UKIRT $K$. The host galaxy is marked in a green circle. The $\mathrm{X}$ ray afterglow position determined by Chandra is marked as a red cross.

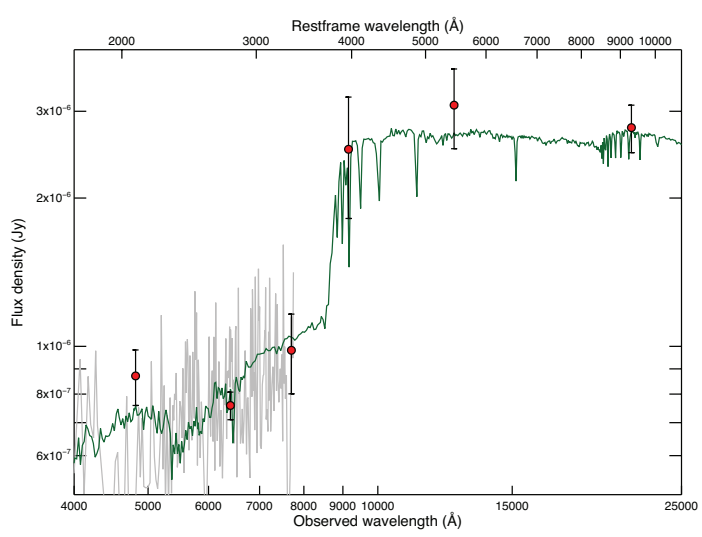

Figure 4: The SED fit to the photometric data ( $g$, $r, i, z, J$ and $K$ ) using the templates of the single stellar populations model [8]. The GTC spectrum is shown in gray.

A significant offset between the center of the host galaxy and the X-ray afterglow has been found for GRB 111117A (see Figure 3). We estimate the offset between the host center and the $\mathrm{X}$-ray afterglow to be $1.0 \pm 0.2$ arcseconds, which corresponding to a distance of $8.4 \pm 1.7 \mathrm{kpc}$ at a redshift of $\mathrm{z}=1.31$. This offset is consistent with the median offset of $5 \mathrm{kpc}$ for short GRBs [9].

Assuming the redshift of 1.31 , the isotropic equivalent $\gamma$-ray energy $\left(E_{\gamma, \text { iso }}\right)$ which is integrated from $1 \mathrm{keV}$ to $10 \mathrm{MeV}$ at the rest-frame is $3.4_{-1.5}^{+5.7} \times 10^{51} \mathrm{erg}$. $E_{\gamma, \text { iso }}$ of GRB $111117 \mathrm{~A}$ is located at the high end of $E_{\gamma \text {,iso }}$ distribution of short GRBs and at the low end of $E_{\gamma, \text { iso }}$ distribution of long GRBs.

\section{References}

[1] Kouveliotou, C., et al., Identification of two classes of gamma-ray bursts, ApJ, 413 (1993) L101

[2] Woosley, S. E., \& Bloom, J. S. The Supernova Gamma-Ray Burst Connection, ARA\&A, 44 (2006) 507

[3] Eichler, D., Livio, M., Piran, T., Schramm, D. M., Nucleosynthesis, neutrino bursts and gamma-rays from coalescing neutron stars, Nature, 340 (1989) 126

[4] Paczynski, B., Cosmological gamma-ray bursts, Acta Astron., 41 (1991) 257

[5] Bromberg, O., Nakar, E., Piran, T., Sari, R., An Observational Imprint of the Collapsar Model of Long Gamma-Ray Bursts, ApJ, 749, 110

[6] Dickey, J. M., Lockman, F. J., H I in the Galaxy, ARA\&A, 28 (1990) 215

[7] Jakobsson, P., et al. Swift Identification of Dark Gamma-Ray Bursts, ApJ, 617 (2004) L21

[8] Maraston, C., Evolutionary population synthesis: models, analysis of the ingredients and application to high-z galaxies, MNRAS, 362 (2005) 799

[9] Fong, W., Berger, E., Fox, D. B., Hubble Space Telescope Observations of Short Gamma-Ray Burst Host Galaxies: Morphologies, Offsets, and Local Environments, ApJ, 708 (2010) 9 